\title{
Study of the Axial Ion Confinement Time for High Particle Flux Operations of GAMMA 10/PDX ${ }^{*)}$
}

\author{
Kazuya ICHIMURA, Yousuke NAKASHIMA, Md. Maidul ISLAM, Md. Shahinul ISLAM, \\ Keita SHIMIZU, Kazuma FUKUI, Masato OHUCHI, Mafumi HIRATA, Ryuya IKEZOE, \\ Shuhei SUMIDA, Masayuki YOSHIKAWA, Naomichi EZUMI, Mizuki SAKAMOTO, \\ Makoto ICHIMURA and Tsuyoshi IMAI \\ Plasma Research center, University of Tsukuba, 1-1-1 Tennoudai, Tsukuba, Ibaraki 305-8577, Japan
}

(Received 30 November 2015 / Accepted 18 February 2016)

\begin{abstract}
Characteristics of axial plasma confinement in recent high-density mode of GAMMA 10/PDX tandem mirror is reported in this paper. The parameter of the end-loss ion flux is an important issue in the divertor simulation studies in GAMMA 10/PDX. With an ICRF heating in the anchor-cells of the machine, large increase in the electron line-density in the central-cell (NLCC) and the end-loss particle flux were observed. The behavior of the electron line density, plasma temperature and the amount of end-loss ion flux during the experiments suggested that the mechanism of the axial ion loss was changed due to the increase of ion collision frequency. In contrast with the standard operations of GAMMA 10/PDX, plasma parameters in recent high-density operations stays in the range of high collision frequency. In such collisional plasma, the end-loss ion particle flux show linear dependence on the plasma density instead of the parabolic dependence expected in plasmas of low collisionfrequency. In the high collision frequency plasma, it is notable that plasma potential $\phi$ largely changes the dependence of the particle flux on the plasma density.
\end{abstract}

(c) 2016 The Japan Society of Plasma Science and Nuclear Fusion Research

Keywords: tandem mirror, GAMMA 10/PDX, mirror confinement, divertor simulation, particle flux

DOI: $10.1585 /$ pfr.11.2405045

\section{Introduction}

Linear machines for plasma experiments are convenient for divertor studies because of their simple structure and operation easiness. The aim of this research is to make it feasible to conduct experiments of divertor plasma physics and plasma wall interaction (PWI) in the range of high plasma temperature relevant to SOL plasma in upcoming ITER or DEMO reactor by using a linear machine. Many linear machines have been contributing to the design of divertor system in fusion experimental machine such as ITER. However, plasma temperature in most linear machines is low because most linear machines do not confine a plasma long enough to store high amount of energy in the plasma. Ion temperature in a typical linear device is less than a few $\mathrm{eV}$, which is much less than that of SOL plasma in fusion reactors, a few hundreds of $\mathrm{eV}$. Therefore, experimental research of linear divertor simulator with high temperature range is required.

A large tandem mirror device, GAMMA 10/PDX is a powerful tool to perform such experimental research since the machine is able to produce the end-loss particle flux which has high temperature [1-3]. In previous research, the energy distribution and the current density of the end-

author'se-mail: ichimara_kazuya@prc.tsukuba.ac.jp

*) This article is based on the presentation at the 25th International Toki Conference (ITC25). loss ion were measured by the end-loss ion energy analyzer (ELIEA) and the particle flux estimated from measured ion current density is $5 \times 10^{22}$ particles $/ \mathrm{m}^{2} \mathrm{sec}$.

In order to increase the amount of particle flux to the value expected in ITER or other fusion device; $10^{23} \sim 10^{24}$ particles $/ \mathrm{m}^{2} \mathrm{sec}$, new antennas of ICRF have been installed and in recent experiments, large increase of plasma density and end-loss particle flux due to the new ICRF heating method were observed [4]. In this article, results of the high-density experiments with new ICRF antenna configurations are reported and discussions on the characteristics of mirror confinement in the newly achieved high-density operations of the tandem mirror are made.

\section{Experimental Setup}

As a feature of the device, GAMMA10/PDX has 27meter machine length and plasma radius larger than $10 \mathrm{~cm}$. As shown in Fig. 1, the machine consists of seven cells: the central cell, anchor cells, plug/barrier cells and end cells. Also, high power (300 kW) ICRF heating systems are equipped and used for plasma production and heating. In a standard operation, $\mathrm{H}_{2}$ plasma is initiated by the ignition of MPD arcjet plasma gun, and heated up to ion temperature of several $\mathrm{keV}$ by ICRF wave from type III (Nagoya type III) and type II (double half turn) antennas. Double arc type (DAT) antennas for ICRF are installed in 


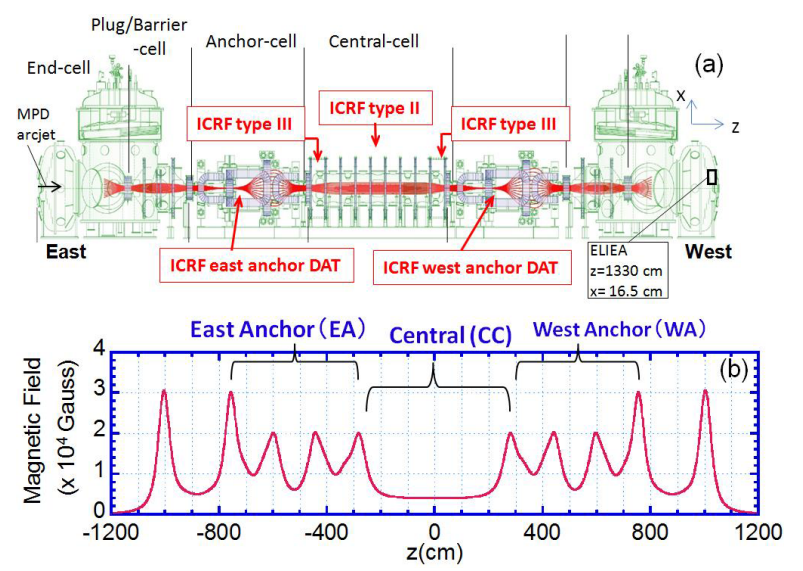

Fig. 1 (a) Vacuum vessels of GAMMA 10/PDX with resonance positions of ICRF heating wave from three antennas (ICRF type III, type II, and anchor DAT) and the position of the end-loss ion energy analyzer. (b) Plot of magnetic field strength along $z$-axis.

both east and west anchor regions and used to heat the plasma in anchor cells. In a series of GAMMA 10/PDX experiments, it was found that adding a plasma heating wave by DAT antenna into an anchor region shows the remarkable effect of increasing the end-loss ion flux [2-4]. Then, in this series, simultaneous heating by both east and west anchor DAT antennas has been performed. As a main diagnostic tool for the end-loss ion flux, end-loss ion energy analyzer (ELIEA) is installed on the inner wall of the end cells in order to observe the energy distribution and the current density of the end-loss ion flux incident to the end region [5].

\section{Results and Discussions}

Figure 2 shows the result of ICRF heating into both east and west anchor cells (EA+WA) [6]. In this case, NLEA, NLWA and NLCC were increased largely and the degree of line density increase during ICRF is almost same in EA and WA. Therefore, symmetry of plasma parameters in east side and west side of the machine can be assumed. On the other hand, ion temperature measured by ELEIA is slightly lowered by ICRF anchor DAT. Degradation of wave heating efficiency due to increase of plasma density is considered to be the cause of lowered ion temperature. In addition, it is notable that the amount of ion particle flux become maximum in the time range from $t=200 \mathrm{~ms}$ to $t$ $=210 \mathrm{~ms}$, where ECRH and NBI is applied to the centralcell. In Fig. 3, the radial profile of the end-loss ion particle flux in the high density experiment is plotted. It seems that the radial profile or plasma radius was enlarged during the additional plasma heating by both east and west anchor DAT antennas. Superimposing of plasma heating by ECRH and NBI for the high density plasma results in an increase and peaking of particle flux.

Ion particle flux measured by west ELIEA plotted

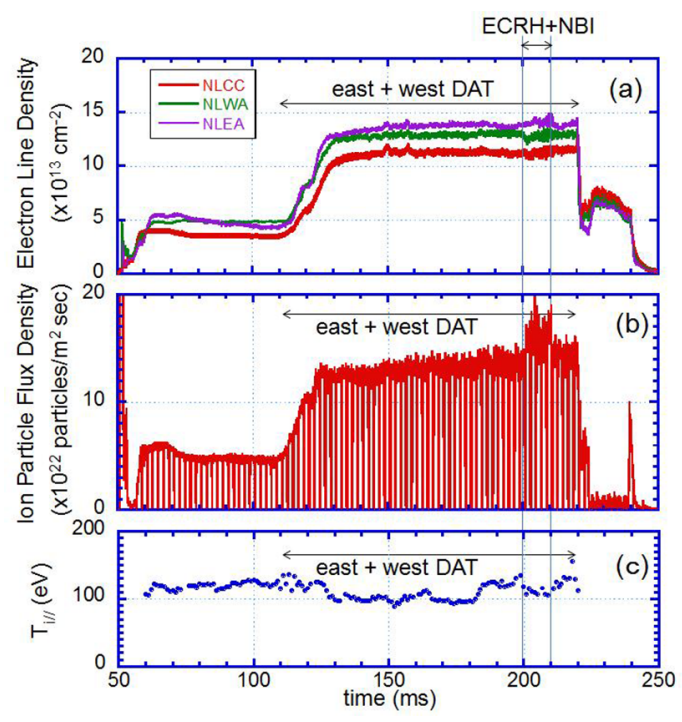

Fig. 2 Results of DAT antenna heating in both east and west anchor cells; (a) electron line densities in each cells, (b) ion particle flux density measured by west-ELIEA and (c) ion temperature of the end-loss ion flux.

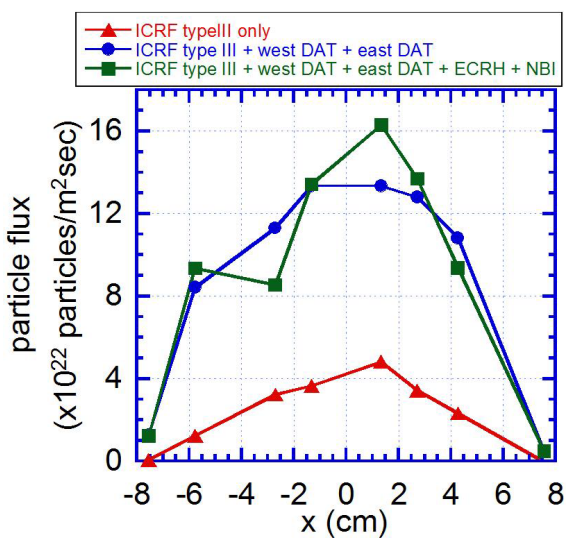

Fig. 3 The ion particle flux measured at the west end-cell plotted against the $x$-axis.

against the electron line density in the central cell (NLCC) in various cases of experiments are shown in Fig. 4 Experiments using ICRF antennas type III and type II, which is a standard experimental situation of GAMMA 10/PDX is represented by solid circles. In the standard condition, the amount of particle flux was relatively small, however, the ion temperature is relatively high in the condition $[2,3]$. Experiments that superimposed the ICRF wave in either east or west anchor regions are plotted as open squares and open circles respectively. In the current experiments, ICRF heating by using west anchor DAT appears slightly more effective compared with east anchor DAT It is considered that the slight asymmetry of the machine in anchor regions is a cause of the difference; however, further investigation is needed. The high-density operations achieved by using both east and west anchor DAT antennas are shown as 


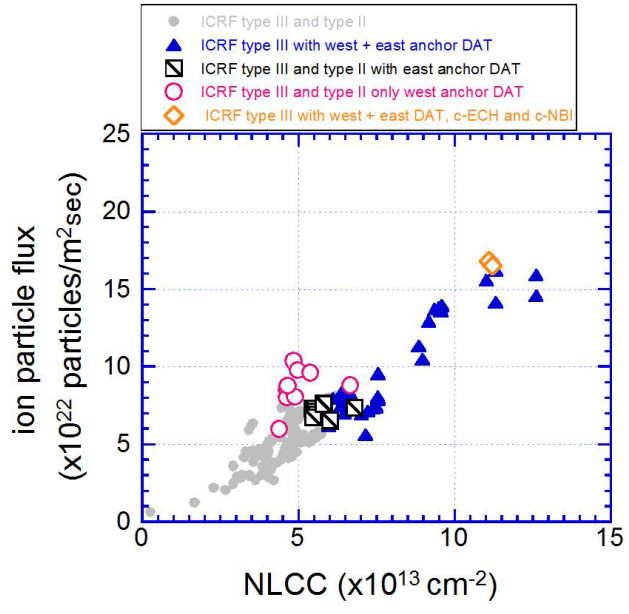

Fig. 4 Ion particle flux measured by ELIEA plotted against the electron line density in the central cell.

solid triangles. In the high density operations, the amount of particle flux is largely enhanced and the value $1.7 \times 10^{23}$ particles $/ \mathrm{m}^{2} \mathrm{sec}$ was achieved. Ion temperature in the operations is relatively low since the strong ion heating from type II antenna is absent in the operation.

Overall, in Fig. 4, there is a co-relationship between the ion particle flux and the electron line density in the central cell. The behavior is natural because the amount of end-loss particle flux is given by the equation $\Gamma_{\mathrm{i}}=$ $N_{\text {total }} / \tau_{\text {ion }}$ where $\Gamma_{\mathrm{i}}$ is an ion particle flux, $\tau_{\text {ion }}$ is the axial ion confinement time and $N_{\text {total }}$ is the total number of ions existing in CC, EA and WA mirror fields. It can be assumed that the addition of plasma heating by anchor DAT antenna increases the amount of particle flux by increasing $N_{\text {total }}$. On the other hand, as seen in Fig. 2, addition of ECRH and NBI increases the particle flux without changing the plasma density. Therefore the effect of ECRH and NBI on the high density plasma can be considered as the change of ion confinement time. The confinement time of GAMMA 10/PDX experiments without confinement potential cases are usually explained by simple-mirror theory [7] written as

$$
\tau_{\text {ion }}=1.8 \times \tau_{\text {ii }} \times \log (R),
$$

where $R$ is the mirror ratio and $\tau_{\mathrm{ii}}$ is the collision time of ion-ion collisions written as

$$
\tau_{\mathrm{ii}}=\frac{6.6 \times 10^{-3} \times T_{\mathrm{i} /}^{\frac{3}{2}}}{N \times\left(10^{-20}\right) \times Z_{\mathrm{i}}^{4} \times \ln \Delta} .
$$

In addition, when considering an actual mirror confined plasma, effect of the plasma potential must be included. The mass of electrons are much smaller than hydrogen ions and therefore electrons in GAMMA 10/PDX escapes from the mirror much faster than ions. As a result, positive electrostatic potential between the mirror confined plasma and the wall of machine is formed. This positive plasma potential works as an enhancement factor of axial ion loss. In equation, the effect of the plasma potential is explained as a degradation of mirror ratio

$$
R_{\mathrm{eff}}=\frac{R}{\left(1+\frac{q \phi}{T_{\mathrm{i} / /}}\right)},
$$

where $\phi$ is plasma potential. The effective mirror ratio $R_{\text {eff }}$ represents the actual mirror confinement performance in experiments.

With considering plasma potential, the dependence of ion particle flux on the plasma density becomes as follows;

$$
\Gamma_{\mathrm{i}} \propto \frac{N^{2}}{\log \left(R_{\mathrm{eff}}\right)} .
$$

Here it can be noted that the particle flux depends on the square of plasma density $N$. However, in Fig. 4, parabolic dependence of the particle flux on the plasma density cannot be seen clearly. Especially in high density operation data indicates that the dependence is linear. This is because the collision frequency in mirror confined plasma became too high and the mechanism of particle loss has been changed. In standard operation of GAMMA 10/PDX, ion-ion collision time is 100 times longer than the average transit time of the ion, $v_{\text {ion }} / L$ where $L$ is the half length of mirror magnetic field. Therefore, few collisions are expected during a transport of end-loss particle to the endregion. On the other hand, collision time is only 1.6 times of the transit time in high density operations and therefore the effect of collision in the loss cone need to be taken into account. This shift of plasma confinement time is theoretically predicted by Monte-carlo simulations [8] and in high collision regime, plasma confinement time can be written as;

$$
\tau_{\text {ion }}=\frac{\sqrt{\pi} \times R_{\mathrm{eff}} \times L}{\sqrt{T_{\mathrm{i} / /}}} .
$$

In this case, the amount of particle flux becomes

$$
\Gamma_{\mathrm{i}} \propto \frac{N \times \sqrt{T_{\mathrm{i} / /}} \times\left(1+\frac{q \phi}{T_{\mathrm{i} / /}}\right) .}{R \times L} .
$$

In high collision plasma, the confinement time is not determined by collision time, but an axial transit time of the ions. Therefore, in high density operations, particle flux is likely to depend linearly on the plasma density.

\section{The Use of Plasma Potential}

According to the theorem of mirror confinement in high collision frequency regime, the particle flux in GAMMA 10/PDX in current high density operations or higher density operations will follow the linear dependence instead of parabolic dependence shown in Fig. 5. That means extremely large power of ICRF heating will be required to achieve particle flux larger than $10^{24}$ 


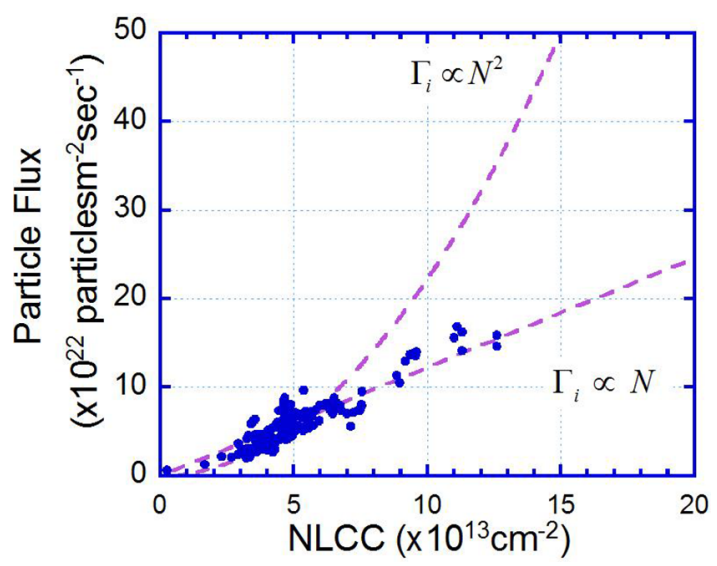

Fig. 5 Ion particle flux measured by ELIEA plotted against the electron line density in the central cell with linear and parabolic fitting curves.

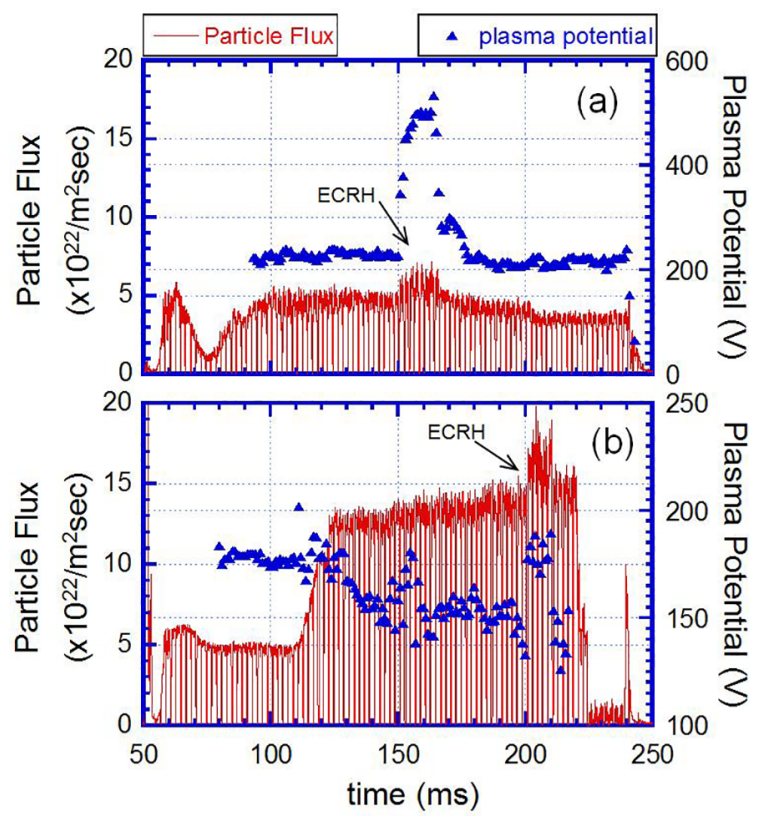

Fig. 6 Time behaviors of ion particle flux and plasma potential in (a) standard operation and (b) high density operation.

particles $/ \mathrm{m}^{2} \mathrm{sec}$. Thus, the use of a plasma potential can be helpful to reduce an effort required to enhance the particle flux. As in equation (1) and (5), mirror confinement time of ions in high density operation depend directly on the mirror ratio $R_{\text {eff }}$ instead of the logarithm of $R_{\text {eff }}$. Therefore the change of mirror ratio due to the plasma potential becomes more effective in high density experiments.

Experimental result of particle flux enhancement by using ECRH is plotted in Figs. 6 (a) and (b). ECRH is an electron heating device and therefore plasma potential is raised by the application of ECRH. Here, in standard collisionless plasma, increase of plasma potential by $200 \mathrm{~V}$ results the increase in particle flux by $0.5 \sim 1.0 \times 10^{22}$ particles $/ \mathrm{m}^{2} \mathrm{sec}$. On the other hand, the increase in plasma density by about $50 \mathrm{~V}$ results the enhancement of particle flux by $3.0 \sim 5.0 \times 10^{22}$ particles $/ \mathrm{m}^{2} \mathrm{sec}$ in high density operation.

\section{Summary}

For the divertor simulation study that is recently focused in GAMMA 10/PDX, the method to enhance the amount of ion particle flux was studied. Plasma heating by new ICRF antennas has effectively enhanced the buildup of plasma density and particle flux. It was observed that the high plasma density and low plasma temperature in the high density operations resulted in the enhancement of ionion collision and changes mirror confinement characteristics. The observation suggested that it will be helpful to use the effect of plasma potential as a support strategy for the future upgrade of the particle flux.

\section{Acknowledgements}

This study was supported by the NIFS as bidirectional collaborative research (NIFS12KUGM066). The author thanks the members of the GAMMA 10 research group for their collaboration on the experiments and for helpful discussions.

[1] Y. Nakashima et al., J. Nucl. Mater. 438, S738 (2013).

[2] K. Ichimura et al., Trans. Fusion Sci. Technol. 63, 1T, 209 (2013).

[3] K. Ichimura et al., Plasma Fusion Res. 7, 2405147 (2012).

[4] R. Ikezoe et al., Fusion Sci. Technol. 68, 63 (2015).

[5] T. Kuwabara et al., Rev. Sci. Instrum. 65, 936 (1994).

[6] S. Sumida et al., Fusion Sci. Technol. 68, 136 (2015).

[7] D. V. Sivukhin, Review of Plasma Physics 4, 202 (1966).

[8] R. Minai et al., J. Phys. Soc. Jpn. 66, 7, 2051 (1997). 